\title{
Studies on the chemical control of luminous bacteria Vibrio harveyi and $V$. splendidus isolated from diseased Penaeus monodon larvae and rearing water
}

\author{
M. C. L. Baticados, C. R. Lavilla-Pitogo, E. R. Cruz-Lacierda, L. D. de la Peña, \\ N. A. Suñaz \\ Fish Health Section, Aquaculture Department, Southeast Asian Fisheries Development Center, Tigbauan, Iloilo 5021, \\ The Philippines
}

\begin{abstract}
The minimum inhibitory concentrations (MICs) and minimum bactericidal concentrations (MBCs) of 24 drugs for luminous bacteria Vibrio harveyi and $V$. splendidus were determined. Only chloramphenicol, sodium nifurstyrenate and the nitrofurans (furazolidone, nitrofurazone, nitrofurantoin and Prefuran) showed relatively low MICs and MBCs $\left(<25 \mu \mathrm{g} \mathrm{ml}{ }^{-1}\right)$. The bacteria showed varied responses to chloramphenicol and Prefuran, and low sensitivity to oxytetracycline. Chloramphenicol, oxytetracycline and Prefuran are commonly used in shrimp hatcheries. Shrimp larvae showed high survival rates and active swimming movement after $24 \mathrm{~h}$ exposure to in vivo bactericidal doses of chloramphenicol, Furacin, nitrofurantoin (protozoea only), oxytetracycline (nauplius only), Prefuran (mysis only) and sodium nifurstyrenate, but the drugs caused deformities in the carapace, rostrum, and setae. Chemical control of luminous vibriosis among shrimp larvae appears limited, based on the efficacy of existing and readily available drugs, because of the possible development of resistant strains of bacteria and the limited tolerance of the shrimp larvae to the drugs.
\end{abstract}

\section{INTRODUCTION}

The occurrence of luminescence in Penaeus monodon larval cultures followed by significant mortality of the larvae have been observed for quite some time in the Province of Aklan in Panay Island, Philippines (Gabasa pers. comm.). It was not until 1987, however, that the microorganisms responsible for this phenomenon were isolated and identified as predominantly Vibrio harveyi and occasionally as $V$. splendidus (Pitogo 1988). Epizootics due to these pathogens in recent years have had devastating effects on the larvae which often suffer mass mortalities. Luminous vibrios are natural inhabitants of the coastal water, and in hatchery systems, eggs (and consequently, larvae) may immediately become infected by the bacteria passed out with the fecal matter of spawners (Pitogo pers. comm.).

The use of drugs in shrimp larval rearing facilities has, more often than not, been the practice in the Philippines (Licop 1988), Taiwan (Jones et al. 1987),
Malaysia, Indonesia (Baticados unpublished), Tahiti (Aquacop 1977), and the United States and its territories (Bell \& Lightner 1987). In the Philippines, the most commonly used drugs are chloramphenicol, oxytetracycline, and Prefuran; despite the presence of the drugs in the water, luminous vibriosis still prevails, indicating the ineffectiveness of the drugs or the dosages used, or perhaps indicating the development of resistance of the luminous vibrios to the drugs. Sunaryanto \& Mariam (1986) reported in vitro sensitivity of luminous bacteria (which they identified as related to Vibrio albensis), isolated from an Indonesian hatchery, to drugs such as chloramphenicol and furazolidone. The present study was performed to determine: (1) the in vitro sensitivity of the luminous bacterial isolates $V$. harveyi and $V$. splendidus to various drugs; (2) the minimum inhibitory concentrations (MICs) and the minimum bactericidal concentrations (MBCs) for drugs to which luminous vibrios are sensitive; and (3) the tolerance of Penaeus monodon larvae to the bactericides. 


\section{MATERIALS AND METHODS}

Sources of bacterial isolates. Luminous bacteria were isolated from Penaeus monodon larvae, rearing water, and seawater in various localities in the provinces of Aklan, Capiz, and Iloilo in Panay Island, Philippines (Table 1). Primary isolation was performed on trypticase soy agar (Lavilla-Pitogo et al. 1990) and stock cultures were kept in nutrient agar (NA) tubes.

Drug sensitivity of luminous bacterial isolates. Antimicrobial sensitivity tests on luminescent vibrios were conducted using newly isolated and identified bacteria. Procedures were based on the standardized disc-agar diffusion method of the National Committee for Clinical Laboratory Standards for antimicrobial susceptibil-

Table 1. Vibrio spp. Luminous vibrios isolated from various sources in different provinces of Panay Island, Philippines. Sources are: $(Z)$ protozoea; $(R W)$ rearing water; $(N)$ nauplius;

(PL) postlarva; (M) mysis; (SW) seawater

\begin{tabular}{|c|c|c|c|}
\hline $\begin{array}{l}\text { Province/ } \\
\text { locality }\end{array}$ & Code no. & Source & Species \\
\hline \multicolumn{4}{|l|}{ Aklan } \\
\hline Batan & Lum 1 & Z & V. harveyi \\
\hline Makato & $\begin{array}{l}\text { B56 } \\
\text { D36 } \\
\text { D39 } \\
\text { D43 } \\
\text { D47 } \\
\text { Lum2 }\end{array}$ & $\begin{array}{l}\text { RW } \\
\text { N } \\
\text { N } \\
\text { PL } \\
\text { PL } \\
M\end{array}$ & $\begin{array}{l}V . \text { harveyi } \\
V . \text { splendidus } \\
V . \text { splendidus } \\
V . \text { harveyi } \\
V . \text { harveyi } \\
V . \text { harveyi }\end{array}$ \\
\hline \multicolumn{4}{|l|}{ New } \\
\hline Washington & $\begin{array}{l}\text { B9 } \\
\text { D8 } \\
\text { D32 } \\
\text { D33 }\end{array}$ & $\begin{array}{l}\text { PL } \\
M \\
\text { PL } \\
\text { PL }\end{array}$ & $\begin{array}{l}V . \text { harveyi } \\
V . \text { harveyi } \\
V . \text { harveyi } \\
V . \text { harveyi }\end{array}$ \\
\hline \multicolumn{4}{|l|}{ Capiz } \\
\hline Pilar & $\begin{array}{l}\text { E6 } \\
\text { E13 } \\
\text { E33 }\end{array}$ & $\begin{array}{l}\text { RW } \\
\text { SW } \\
\text { PL }\end{array}$ & $\begin{array}{l}V . \text { harveyi } \\
V . \text { harveyi } \\
V . \text { harveyi }\end{array}$ \\
\hline Roxas & $\begin{array}{l}\text { E2 } \\
\text { E45 } \\
\text { LBS24 } \\
\text { LBS25 }\end{array}$ & $\begin{array}{l}\text { SW } \\
\text { PL } \\
N \\
N\end{array}$ & $\begin{array}{l}\text { V. harveyi } \\
V . \text { harveyi } \\
V . \text { splendidus } \\
V . \text { splendidus }\end{array}$ \\
\hline \multicolumn{4}{|l|}{ Iloilo } \\
\hline Leganes & $\begin{array}{l}\text { LBS1 } \\
\text { LBS12 }\end{array}$ & $\begin{array}{l}\text { SW } \\
M\end{array}$ & $\begin{array}{l}\text { V. harveyi } \\
\text { V. harveyi }\end{array}$ \\
\hline Oton. & $\begin{array}{l}\text { O2 } \\
\text { OPL2 } \\
\text { ORW } \\
\text { OZ2 }\end{array}$ & $\begin{array}{l}\text { SW } \\
\text { PL } \\
\text { RW } \\
Z\end{array}$ & $\begin{array}{l}V . \text { harveyi } \\
V . \text { harveyi } \\
V . \text { harveyi } \\
V . \text { harveyi }\end{array}$ \\
\hline Tigbauan & $\begin{array}{l}\text { JJM2-7 } \\
\text { Phy3 } \\
\text { Phy4 } \\
\text { TRS14 }\end{array}$ & $\begin{array}{l}\mathrm{M} \\
\mathrm{RW} \\
\mathrm{RW} \\
\mathrm{SW}\end{array}$ & $\begin{array}{l}V . \text { harveyi } \\
V . \text { harveyi } \\
V . \text { harveyi } \\
V . \text { harveyi }\end{array}$ \\
\hline
\end{tabular}

ity tests (Finegold \& Martin 1982). Each isolate was subcultured in NA for 12 to $18 \mathrm{~h}$, from which 5 to 10 colonies were taken and emulsified in brain heart infusion broth (BHIB). The BHIB culture was incubated for 2 to $3 \mathrm{~h}$ and adjusted to $0.5 \mathrm{McF}$ arland's Barium Sulfate Standard solution (corresponding to $1.5 \times 10^{8} \mathrm{CFU}$ $\mathrm{ml}^{-1}$ ) with sterile seawater The standardized culture was swabbed thoroughly on Mueller-Hinton agar which was then dried for 30 to $60 \mathrm{~min}$ before sensitivity discs (Baltimore Biological Laboratories, BBL, discs, $6 \mathrm{~mm}$ diameter, unless specified) were placed on each quadrant of the plate. The zones of inhibition were measured after 12 to $18 \mathrm{~h}$ incubation. Test organisms used for quality control were Escherichia coli (ATCC 25922), Staphylococcus aureus (ATCC 29213), and Pseudomonas aeruginosa (ATCC 27853).

Further tests for the MICs and MBCs were conducted using a modified version of Finegold \& Martin's (1982) tube dilution method. Weighed samples of 24 drugs were dissolved in distilled water and diluted to obtain working solutions of $100 \mu \mathrm{g} \mathrm{ml}^{-1}$. Decreasing amounts of the drug solutions were added to a series of sterile $13 \times 100 \mathrm{~mm}$ screw-capped tubes containing increasing amounts of the diluent, Mueller-Hinton broth. The bacterial isolates, emulsified in BHIB, were standardized with $0.5 \mathrm{McF}$ arland's Barium Sulfate Standard solution and diluted $1 \times 10^{4}$ with sterile seawater to obtain a $1 \times 10^{4} \mathrm{CFU} \mathrm{m}{ }^{-1}$ concentration of inoculum. The density of the inoculum was based on the density of luminous vibrios often found in shrimp larvae and rearing water during disease outbreaks (Lavilla-Pitogo et al. 1990). One ml of this diluted bacterial culture was added to each of the tubes, except the last tube which served as the control (see example in Table 2). Two replicates per concentration were used for two trials per isolate.

The tubes were incubated for 18 to $24 \mathrm{~h}$ after which they were examined visually for turbidity, i.e. bacterial growth. The lowest concentration of the drug in the series that showed no growth was considered the MIC

Table 2. In vitro testing of antimicrobial agents using a modified tube dilution method. Diluent $=$ Mueller-Hinton broth

\begin{tabular}{ccccc|}
\hline $\begin{array}{c}\text { Tube } \\
\text { no. }\end{array}$ & $\begin{array}{c}\text { Diluent } \\
\text { (ml) }\end{array}$ & $\begin{array}{c}\text { Drug } \\
(\mathrm{ml})\end{array}$ & $\begin{array}{c}\text { Diluted } \\
\text { culture } \\
(\mathrm{ml})\end{array}$ & $\begin{array}{c}\text { Final drug } \\
\text { concentration, } \\
\left.(\text { (ug ml })^{-1}\right)\end{array}$ \\
\hline 1 & 0 & 1.0 & 1 & 50 \\
2 & 0.5 & 0.5 & 1 & 25 \\
3 & 0.6 & 0.4 & 1 & 20 \\
4 & 0.8 & 0.2 & 1 & 10 \\
5 & 0.9 & 0.1 & 1 & 5 \\
6 & 0.98 & 0.02 & 1 & 1 \\
7 & 1 & 0 & 1 & $0(+$ control) \\
8 & 2 & 0 & 0 & $0(-$ control) \\
\hline
\end{tabular}


To determine the $\mathrm{MBC}, 0.1 \mathrm{ml}$ from each tube that showed no turbidity was spread on NA plates in 2 replicates. Plates were incubated for 18 to $24 \mathrm{~h}$ after which they were examined for the presence of luminous bacterial colonies. Colonies were enumerated by direct counting, and the lowest concentration of the drug that resulted in no growth was considered the $\mathrm{MBC}$.

Incubation temperatures for all cultures were around 26 to $28^{\circ} \mathrm{C}$.

Tolerance tests. The tolerance of nauplii, protozoeae, and myses to concentrations double the MICs and MBCs of the 7 drugs were determined in a 24 h static bioassay procedure. Chloramphenicol, Furacin, nitrofurantoin, nitrofurazone, oxytetracycline, Prefuran, and sodium nifurstyrenate $(10 \%)$ were chosen based on high sensitivity of the isolates to the drugs, availability, and/or relatively low cost. Shrimp larvae were stocked in 8-l glass aquaria at a density of 10 ind. $1^{-1}$. The aquaria were covered with a black cloth to minimize photodegradation of chemicals. The temperature and salinity ranges during the experiment were 25.5 to $28^{\circ} \mathrm{C}$ and 32 to $34 \%$, respectively. Larvae were not fed during tolerance tests because excess feed and fecal matter in the aquaria might have reduced the availability of the drugs. The effects of the drugs on the survival, swimming movement, and morphology of larvae were determined at the end of the experiment.

The bioassay was conducted using a completely randomized design with at least 2 drug concentrations $(2 \times$ $\mathrm{MIC}$ and $2 \times \mathrm{MBC}$ ) and 3 replicates per concentration. Results were analyzed using analysis of variance (ANOVA) and least significant difference (LSD) after arc sine transformation of the percent values.

\section{RESULTS}

\section{Drug sensitivity of luminous bacterial isolates}

Results of the antimicrobial sensitivity tests using the disc-agar diffusion method are shown in Table 3. After incubation, mutant strains of bacteria were evident as isolated colonies in the clear zones of inhibition. This was particularly true for bacteria exposed to chloramphenicol, novobiocin, oxytetracycline, and polymyxin B.

Table 4 summarizes the results in Table 3 in terms of percent susceptible bacterial isolates. High sensitivities (> $50 \%$ of bacterial isolates susceptible) to chloramphenicol, furazolidone, nalidixic acid, nitrofurantoin, nitrofurazone, and tetracycline were noted. It was also apparent that the isolates were resistant to erythromycin, kanamycin, penicillin $G$, and streptomycin. The remaining drugs had little effect on the majority of the isolates, exerting inhibitory or killing effects on less than $30 \%$ of isolates tested.

The MICs and MBCs of the drugs tested on several luminous bacterial isolates are shown in Table 5. Only chloramphenicol, sodium nifurstyrenate, and the nitrofurans (furazolidone, nitrofurazone, nitrofurantoin and Prefuranj showed generally low MICs and MBCs $\left(<25 \mu \mathrm{g} \mathrm{ml}^{-1}\right)$. Responses of some isolates to specific drugs, e.g. chloramphenicol and Prefuran, also varied widely.

\section{Tolerance tests}

Results of the tolerance tests on nauplii, protozoeae, and myses to chloramphenicol, Furacin, nitrofurantoin, nitrofurazone, oxytetracycline, Prefuran and sodium nifurstyrenate are shown in Table 6. Based on survival rates and behavior, nauplii could tolerate $10 \mathrm{mg} \mathrm{l}^{-1}$ chloramphenicol, $50 \mathrm{mg} \mathrm{l}^{-1}$ Furacin, $4 \mathrm{mg} \mathrm{l}^{-1}$ nitrofurazone, $200 \mathrm{mg} \mathrm{l}^{-1}$ oxytetracycline, $2 \mathrm{mg} \mathrm{l}^{-1}$ Prefuran and $20 \mathrm{mg}^{-1}$ sodium nifurstyrenate. Protozoeae could tolerate $10 \mathrm{mg} \mathrm{l}^{-1}$ chloramphenicol, $50 \mathrm{mg} \mathrm{l}^{-1}$ Furacin, $30 \mathrm{mg} \mathrm{l}^{-1}$ nitrofurantoin, $2 \mathrm{mg} \mathrm{l}^{-1}$ Prefuran, and $20 \mathrm{mg}$ $1^{-1}$ sodium nifurstyrenate; while myses could tolerate $100 \mathrm{mg} \mathrm{l}^{-1}$ chloramphenicol, $50 \mathrm{mg} \mathrm{l}^{-1}$ Furacin, $4 \mathrm{mg}$ $\mathrm{l}^{-1}$ nitrofurazone, $100 \mathrm{mg} \mathrm{l}^{-1}$ oxytetracycline, $4 \mathrm{mg} \mathrm{l}^{-1}$ Prefuran, and $40 \mathrm{mg} \mathrm{l}^{-1}$ sodium nifurstyrenate. In most cases, however, morphological deformities such as spread carapaces, bent rostrums, and curled setae were observed among drug-exposed larvae with fairly high survival rates.

\section{DISCUSSION}

The luminous vibrio isolates were apparently sensitive to chloramphenicol, nalidixic acid, tetracycline, and the nitrofurans, but were particularly resistant to erythromycin, kanamycin, penicillin, and streptomycin. Likewise, luminous vibrios isolated in Indonesia were found sensitive to chloramphenicol (20 $\left.\mathrm{mg} \mathrm{l}^{-1}\right)$, furazolidone (10 $\mathrm{mg} \mathrm{l}^{-1}$ ) and Prefuran $\left(1 \mathrm{mg} \mathrm{l}^{-1}\right)$, but were resistant to kanamycin (Sunaryanto \& Mariam 1986).

Chloramphenicol is a broad-spectrum bacteriostatic antibiotic that is easily absorbed through the gastrointestinal tract of mammals (Aronson \& Kirk 1974 in Nusbaum \& Shotts 1981) and attains blood concentrations that could be considered therapeutic (Lasserne 1972 in Colorni \& Paperna 1983). Although shrimp nauplii and protozoeae may survive chloramphenicol well at $50 \mathrm{mg} \mathrm{l}^{-1}$ for $24 \mathrm{~h}$, its use may lead to physical deformities and possible development of bacterial resistance. Furthermore, chloramphenicol has erythrocyte destroying effects in humans (Farkas et al. 1982) 
Table 3. Vibrio spp. Sensitivity of luminous vibrio isolates to various antimicrobial agents Antimicrobial agents are: (A) Ampicillin, $30 \mu g_{i}(C 30)$ chloramphenicol, $30 \mu g_{i}(C 100)$ chloramphenicol, $100 \mu \mathrm{g}$; (E) erythromycin, $15 \mu g_{;}(F)$ furazolidone, $100 \mu g ;(K)$ kanamycin, $30 \mu g_{i}(N)$ nalidixic acid, $30 \mu g_{i}$ (FM) nitrofurantoin, $100 \mu g_{i}$ (FC) nitrofurazone, $100 \mu g_{i}$ (NB30) novobiocin, $30 \mu g_{i}$ (OT) oxytetracycline, $5 \mu \mathrm{g}$; (P) penicillin $\mathrm{G}, 10$ units $(\mathrm{PB})$ polymyxin $\mathrm{B}, 300$ units; (S) streptomycin, $10 \mu \mathrm{g}$; (SD) sulfadiazine, 25 ug; (T) tetracycline, $200 \mu g$; (SSS) triple sulfa, $250 \mu \mathrm{g}$. Reactions are: (NT) not tested; (R) resistant; (S) susceptible; (I) intermediate

\begin{tabular}{|c|c|c|c|c|c|c|c|c|c|c|c|c|c|c|c|c|c|}
\hline $\begin{array}{l}\text { Isolate } \\
\text { code no. }\end{array}$ & $\mathrm{A}$ & C 30 & $\mathrm{C} 100$ & $\mathrm{E}$ & $F$ & $K$ & $N$ & $\begin{array}{l}\text { Antim } \\
\text { FM }\end{array}$ & $\begin{array}{c}\text { crobi } \\
\text { FC }\end{array}$ & $\begin{array}{l}\text { agents } \\
\text { NB30 }\end{array}$ & OT & $\mathrm{P}$ & $\mathrm{PB}$ & S & $\mathrm{SD}$ & $\mathrm{T}$ & SSS \\
\hline Lum 1 & NT & $R$ & NT & $\mathrm{R}$ & $\mathrm{S}$ & I & NT & $S$ & $\mathrm{~S}$ & I & $\mathrm{R}$ & $\mathrm{R}$ & I & $\mathrm{R}$ & $\mathrm{R}$ & $N T$ & $\mathrm{R}$ \\
\hline B56 & NT & $\mathrm{R}$ & NT & $\mathrm{R}$ & $\mathrm{S}$ & $\mathrm{R}$ & NT & I & $\mathrm{S}$ & $\mathrm{R}$ & $\mathrm{R}$ & $\mathrm{R}$ & I & $\mathrm{R}$ & $\mathrm{R}$ & $N T$ & $\mathrm{R}$ \\
\hline D36 & $\mathrm{S}$ & $\mathrm{S}$ & $\mathrm{S}$ & $\mathrm{I}$ & $\mathrm{S}$ & $\mathrm{R}$ & $\mathrm{S}$ & $\mathrm{S}$ & $\mathrm{S}$ & $\mathrm{S}$ & I & $\mathrm{R}$ & $\mathrm{S}$ & I & $\mathrm{R}$ & $S$ & $\mathrm{R}$ \\
\hline D39 & $\mathrm{R}$ & $\mathrm{s}$ & $S^{\circ}$ & I & I & $\mathrm{R}$ & NT & $\mathrm{S}$ & I & I & $\mathrm{R}$ & $\mathrm{R}$ & NT & $\mathrm{R}$ & $\mathrm{R}$ & $\mathrm{S}$ & $\mathrm{R}$ \\
\hline D47 & $\mathrm{R}$ & $\mathrm{S}$ & S & $\mathrm{R}$ & I & $\mathrm{R}$ & NT & I & $\mathrm{S}$ & I & I & $\mathrm{R}$ & $\mathrm{R}$ & $\mathrm{R}$ & $\mathrm{R}$ & $\mathrm{S}$ & $R$ \\
\hline Lum2 & NT & $\mathrm{R}$ & NT & I & $\mathrm{S}$ & $\mathrm{R}$ & NT & I & $\mathrm{S}$ & $\mathrm{R}$ & $\mathrm{R}$ & $\mathrm{R}$ & $\mathrm{R}$ & $\mathrm{R}$ & $R$ & NT & $\mathrm{R}$ \\
\hline B9 & NT & $\mathrm{R}$ & $N T$ & $\mathrm{R}$ & $\mathrm{S}$ & 1 & NT & I & $\mathrm{S}$ & $\mathrm{R}$ & $\mathrm{R}$ & $\mathrm{R}$ & $\mathrm{R}$ & $\mathrm{R}$ & $\mathrm{R}$ & NT & $\mathrm{R}$ \\
\hline D8 & $\mathrm{R}$ & $\mathrm{S}$ & $\mathrm{S}$ & I & I & $\mathrm{R}$ & $\mathrm{S}$ & $\mathrm{S}$ & $\mathrm{S}$ & r & $\mathrm{R}$ & $\mathrm{R}$ & $\mathrm{R}$ & $\mathrm{R}$ & $\mathrm{R}$ & $\mathrm{S}$ & I \\
\hline D32 & $\mathrm{R}$ & $\mathrm{S}$ & $\mathrm{S}$ & I & $\mathrm{S}$ & $\mathrm{R}$ & $S$ & $S$ & $\mathrm{~S}$ & $\mathrm{I}^{*}$ & I & $\mathrm{R}$ & $I^{*}$ & $\mathrm{R}$ & $\mathrm{R}$ & $\mathrm{S}$ & I \\
\hline D33 & $\mathrm{R}$ & $\mathrm{S}$ & $\mathrm{S}$ & I & $S$ & $\mathrm{R}$ & NT & I & $\mathrm{S}$ & $\mathrm{R}$ & $\mathrm{R}$ & $\mathrm{R}$ & $\mathrm{R}$ & $\mathrm{R}$ & $\mathrm{R}$ & $\mathrm{S}$ & $\mathrm{R}$ \\
\hline $\mathrm{E} 6$ & $\mathrm{R}$ & $\mathrm{S}$ & $S$ & $\mathrm{R}$ & I & $\mathrm{R}$ & $N T$ & i & $\mathrm{s}$ & $\mathrm{R}$ & $\mathrm{R}$ & $\mathrm{R}$ & $\mathrm{R}$ & $\mathrm{R}$ & $R$ & $S$ & $\mathrm{R}$ \\
\hline E13 & $R$ & $\mathrm{~S}$ & $\mathrm{~S}$ & $\mathrm{R}$ & $\mathrm{S}$ & I & S & $\mathrm{S}$ & $\mathrm{S}$ & I & $\mathrm{R}$ & $\mathrm{R}$ & $\mathrm{S}^{*}$ & $\mathrm{R}$ & $\mathrm{R}$ & $\mathrm{S}$ & $\mathrm{R}$ \\
\hline E33 & $\mathrm{R}$ & $\mathrm{R}$ & $\mathrm{R}$ & $\mathrm{R}$ & I & $\mathrm{R}$ & NT & S & $\mathrm{S}$ & $\mathrm{R}$ & I & $\mathrm{R}$ & $\mathrm{R}$ & $\mathrm{R}$ & $\mathrm{R}$ & $\mathrm{S}$ & $\mathrm{R}$ \\
\hline E2 & $\mathrm{R}$ & $\mathrm{S}$ & $\mathrm{S}$ & $\mathrm{R}$ & I & $\mathrm{R}$ & $\mathrm{S}$ & I & $\mathrm{S}$ & $\mathrm{R}$ & I & $\mathrm{R}$ & $\mathrm{R}$ & $\mathrm{R}$ & $\mathrm{R}$ & $S$ & $\mathrm{R}$ \\
\hline E45 & $\mathrm{S}$ & $\mathrm{S}$ & S & $\mathrm{R}$ & $\mathrm{S}$ & $\mathrm{R}$ & $\mathrm{S}$ & I & $\mathrm{S}$ & $\mathrm{S}$ & 1 & $\mathrm{R}$ & $\mathrm{S}$ & $\mathrm{R}$ & $\mathrm{R}$ & $S$ & I \\
\hline LBS 24 & $\mathrm{~S}$ & $\mathrm{~s}$ & $\mathrm{~S}$ & $\mathrm{R}$ & I & $\mathrm{R}$ & NT & I & $\mathrm{S}$ & $\mathrm{R}$ & $\mathrm{R}$ & $\mathrm{R}$ & I & $\mathrm{R}$ & $\mathrm{R}$ & $\mathrm{S}$ & $\mathrm{S}$ \\
\hline LBS25 & $\mathrm{S}$ & $\mathrm{S}$ & $\mathrm{S}$ & I & I & $\mathrm{R}$ & $S$ & S & $\mathrm{S}$ & $\mathrm{R}$ & $\mathrm{S}$ & $\mathrm{R}$ & I & $\mathrm{R}$ & $\mathrm{S}$ & $\mathrm{S}$ & I \\
\hline LBS1 & $\mathrm{R}$ & $\mathrm{R}$ & $\mathrm{R}$ & $\mathrm{R}$ & $\mathrm{R}$ & $\mathrm{R}$ & $\mathrm{S}$ & $\mathrm{S}$ & $S$ & I & $\mathrm{R}$ & $\mathrm{R}$ & NT & $\mathrm{R}$ & $\mathrm{R}$ & $S$ & $\mathrm{R}$ \\
\hline LBS12 & $\mathrm{R}$ & $\mathrm{S}$ & $\mathrm{S}$ & $\mathrm{R}$ & $\mathrm{S}$ & I & NT & $\mathrm{S}$ & $\mathrm{s}$ & $\mathrm{S}^{\circ}$ & $I^{*}$ & $\mathrm{R}$ & $\mathrm{R}$ & $\mathrm{R}$ & $\mathrm{R}$ & $S$ & $\mathrm{R}$ \\
\hline $\mathrm{O} 2$ & NT & $\mathrm{R}$ & NT & $R$ & I & $\mathrm{R}$ & NT & I & $\mathrm{S}$ & $\mathrm{R}$ & $\mathrm{R}$ & $\mathrm{R}$ & 1 & $R$ & $\mathrm{R}$ & NT & $\mathrm{R}$ \\
\hline OPL2 & $N T$ & $R$ & NT & NT & NT & NT & NT & $\mathrm{S}$ & $S$ & NT & $\mathrm{S}^{*}$ & NT & NT & NT & NT & NT & NT \\
\hline ORW & NT & $\mathrm{R}$ & NT & NT & NT & NT & NT & $S$ & $S$ & $N T$ & $\mathrm{~S}$ & NT & NT & NT & NT & NT & NT \\
\hline $\mathrm{OZ2}$ & $\mathrm{R}$ & $\mathrm{R}$ & $\mathrm{s}$ & $\mathrm{R}$ & $S$ & I & $\mathrm{NT}$ & $\mathrm{S}$ & $\mathrm{S}$ & $\mathrm{s}$ & $I^{\prime}$ & $\mathrm{R}$ & $\mathrm{R}$ & $\mathrm{R}$ & $\mathrm{R}$ & $\mathrm{S}$ & $\mathrm{R}$ \\
\hline JJM2 & $\mathrm{R}$ & $\mathrm{R}$ & $\mathrm{R}$ & I & I & $\mathrm{R}$ & NT & $\mathrm{R}$ & I & $R$ & $\mathrm{R}$ & $R$ & $\mathrm{~S}$ & $\mathrm{R}$ & $\mathrm{R}$ & $S$ & $R$ \\
\hline Phy 3 & $\mathrm{R}$ & $\mathrm{R}$ & I & $\mathrm{R}$ & $S$ & $\mathrm{R}$ & NT & $\mathrm{S}$ & $\mathrm{S}$ & $\mathrm{R}$ & $\mathrm{R}$ & $\mathrm{R}$ & $\mathrm{R}$ & $\mathrm{R}$ & $R$ & $\mathrm{~S}$ & $\mathrm{R}$ \\
\hline Phy 4 & $\mathrm{R}$ & $\mathrm{S}$ & $S$ & I & $\mathrm{S}$ & $\mathrm{J}$ & $\mathrm{S}$ & $\mathrm{S}$ & $\mathrm{S}$ & $\mathrm{S}$ & $\mathrm{S}$ & $\mathrm{R}$ & $\mathrm{S}$ & I & $\mathrm{R}$ & $\mathrm{S}$ & $\mathrm{R}$ \\
\hline TRS14 & $\mathrm{S}$ & $\mathrm{R}$ & $\mathrm{S}$ & I & I & $\mathrm{R}$ & $\mathrm{S}$ & $\mathrm{S}$ & $\mathrm{S}$ & $\mathrm{S}$ & $\mathrm{S}$ & $\mathrm{R}$ & I & $R$ & $\hat{S}$ & $\mathrm{~S}$ & $\mathrm{~S}$ \\
\hline
\end{tabular}

and may be harmful to users who come in contact with it (USFDA 1986d and Schnick 1987 in Williams \& Lightner 1988), so it is unlikely that the drug would ever be approved for use by any drug control board of any country.

A very important consideration in the use of chemotherapy in aquaculture is economics, i.e. the value of the drug treatment must always be equated with the value of the stock (Poupard 1978). The high MBCs obtained for nalidixic acid and oxytetracycline would mean the use of large quantities of the drugs, i.e. $>200$ and $>100 \mathrm{~g} \mathrm{ton}^{-1}$, respectively, assuming that the larvae could tolerate the required levels. On a per $100 \mathrm{~g}$ basis, nalidixic acid costs $2.6 \times$ more than chloramphenicol, $2.4 \times$ more than furazolidone, $6.4 \times$ more than nitrofurantoin, $11.7 \times$ more than nitrofurazone, 3.2 $\times$ more than oxytetracycline (SIGMA 1989), $1.1 \times$ more than Prefuran, and $6.7 \times$ more than sodium nifurstyrenate. Thus, the prohibitive cost of treatment using nalidixic acid and oxytetracycline would be particularly disadvantageous and their use would not be economically feasible - even if all isolates were found to be susceptible to the drugs.

Oxytetracycline is the most widely applied antibiotic in finfish culture and is fast gaining popularity in shrimp culture, although its use on shrimps has yet to be approved by the U.S. Food and Drug Administration (Williams \& Lightner 1988). The drug had been used for over a decade in Hungary, then lost its effectiveness because of development of resistance against it (Farkas et al. 1982). Oxytetracycline reportedly enhanced the production of plasmid-mediated resistance in aquatic bacteria (Shotts et al. 1976 in Nusbaum \& Shotts 1981).

It is also likely that formation of bacterial resistance would be rapid in the case of nitrofurans because they are persistent in water (Beladi et al. 1978 in Farkas et al. 1982). Nifurpirinol (the active ingredient of Prefuran) and nitrofurazone were found to retain their anti- 
Table 4. Vibrio spp. Summary of antimicrobial sensitivity of luminous vibrios using the disc-agar diffusion method

\begin{tabular}{|c|c|c|c|c|c|c|c|}
\hline \multirow[t]{2}{*}{ Compound } & \multirow{2}{*}{$\begin{array}{c}\text { Disc } \\
\text { content }^{\mathrm{a}}\end{array}$} & \multirow{2}{*}{$\begin{array}{c}\text { Zone diam. (mm) } \\
\text { indicating } \\
\text { susceptibility }\end{array}$} & \multirow{2}{*}{$\begin{array}{l}\text { Total } \\
\text { isolates } \\
\text { tested }\end{array}$} & \multicolumn{4}{|c|}{ Isolates susceptible $(\%)$} \\
\hline & & & & Aklan & Capiz & Iloilo & Panay Island \\
\hline Ampicillin & $30 \mu \mathrm{g}$ & $14^{\mathrm{b}}$ & 20 & 17 & 43 & 14 & 25 \\
\hline \multirow{2}{*}{ Chloramphenicol } & $30 \mu \mathrm{g}$ & 18 & 27 & 60 & 86 & 20 & 52 \\
\hline & $100 \mu \mathrm{g}^{\mathrm{c}}$ & 21 & 20 & 100 & 86 & 57 & 80 \\
\hline Erythromycin & $15 \mu \mathrm{g}$ & 23 & 25 & 0 & 0 & 0 & 0 \\
\hline Furazolidone & $100 \mu \mathrm{g}$ & $15^{d}$ & 25 & 70 & 29 & 50 & 52 \\
\hline Kanamycin & $30 \mu \mathrm{g}$ & 18 & 25 & 0 & 0 & 0 & 0 \\
\hline Nalidixic acid & $30 \mu \mathrm{g}$ & $19^{\mathrm{e}}$ & 10 & 100 & 100 & 100 & 100 \\
\hline Nitrofurantoin & $100 \mu \mathrm{g}$ & $17^{\mathrm{e} . \mathrm{f}}$ & 27 & 50 & 43 & 80 & 59 \\
\hline Nitrofurazone & $100 \mu \mathrm{g}$ & $16^{9}$ & 27 & 90 & 100 & 90 & 93 \\
\hline Novobiocin & $30 \mu \mathrm{g}$ & 22 & 25 & 10 & 14 & 50 & 24 \\
\hline Oxytetracycline & $5 \mu \mathrm{g}$ & $19^{h}$ & 27 & 0 & 14 & 40 & 18 \\
\hline Penicillin $G$ & $10 \mathrm{U}$ & 22 & 25 & 0 & 0 & 0 & 0 \\
\hline Polymyxin B & $300 \mathrm{U}$ & 12 & 23 & 11 & 29 & 29 & 22 \\
\hline Streptomycin & $10 \mu \mathrm{g}$ & 15 & 25 & 0 & 0 & 0 & 0 \\
\hline Sulfadiazine & $0.25 \mathrm{mg}$ & $17^{\mathrm{i}}$ & 25 & 0 & 14 & 12 & 8 \\
\hline Tetracycline & $200 \mu \mathrm{g}^{\mathrm{c}}$ & 16.5 & 20 & 100 & 100 & 100 & 100 \\
\hline Triple sulfa & $250 \mu \mathrm{g}$ & 17 & 25 & 0 & 14 & 12 & 8 \\
\hline \multicolumn{8}{|c|}{ a BBL (Baltimore Biological Laboratories) discs, $6 \mathrm{~mm}$ diam. } \\
\hline \multicolumn{8}{|c|}{ Based on BBL disc with $10 \mu \mathrm{g}$ Ampicillin } \\
\hline \multicolumn{8}{|c|}{ 'Showa disc, $8 \mathrm{~mm}$ diam. } \\
\hline \multicolumn{8}{|c|}{ d Based on Showa disc with $20 \mathrm{~g}$ Furazolidone } \\
\hline \multicolumn{8}{|c|}{ e Based on susceptibility data of organisms isolated from human urinary tract infection } \\
\hline \multicolumn{8}{|c|}{ 'Based on BBL disc with $300 \mathrm{~g}$ Nitrofurantoin } \\
\hline \multicolumn{8}{|c|}{${ }^{\mathrm{g}}$ From Ford et al. (1986) } \\
\hline \multicolumn{8}{|c|}{ hased on BBL disc with $30 \mathrm{~g}$ Tetracycline } \\
\hline${ }^{1}$ From Bactosensit & SCS, Difco L & aboratories 1984 & iord et & $\mu \mathrm{g} s$ & & & \\
\hline
\end{tabular}

Table 5. Vibrio spp. Bacteriostatic (S) and bactericidal (C) concentrations ( $\mu \mathrm{g} \mathrm{ml}^{-1}$ ) of antibacterial compounds tested on several luminous vibrio isolates

\begin{tabular}{|c|c|c|c|c|c|c|c|c|c|c|c|c|c|c|}
\hline \multirow{3}{*}{$\begin{array}{l}\text { Antibacterial } \\
\text { compounds }\end{array}$} & \multicolumn{14}{|c|}{ Luminous vibrio isolates } \\
\hline & \multicolumn{2}{|c|}{ D8 } & \multicolumn{2}{|c|}{ D33 } & \multicolumn{2}{|c|}{ D39 } & \multicolumn{2}{|c|}{ JJM2 } & \multicolumn{2}{|c|}{ LBS1. } & \multicolumn{2}{|c|}{ LBS24 } & \multicolumn{2}{|c|}{ Phy3 } \\
\hline & $\mathrm{S}$ & $\mathrm{C}$ & $\mathrm{S}$ & $\mathrm{C}$ & $S$ & C & $\mathrm{S}$ & $C$ & S & C & $\mathrm{s}$ & $\mathrm{C}$ & $S$ & $\mathrm{C}$ \\
\hline Argentyne & 100 & $>100$ & 100 & $>100$ & 100 & $>100$ & 100 & $>100$ & 100 & $>100$ & 100 & $>100$ & 100 & $>100$ \\
\hline Chloramine $\mathrm{T}$ & $<50$ & 50 & $<50$ & 50 & $<50$ & 50 & $<50$ & 50 & $<50$ & 50 & $<50$ & 50 & $<50$ & 50 \\
\hline Chloramphenicol & 2 & 3 & 3 & 4 & NT & NT & 50 & $>50$ & 50 & $>50$ & 3 & 25 & 25 & $>50$ \\
\hline Chlorine & 45 & 50 & 42 & 45 & NT & NT & 40 & 45 & 45 & 50 & NT & NT & 40 & 40 \\
\hline Cu Sulfate & $<50$ & 50 & $<50$ & 50 & 50 & $>50$ & 50 & $>50$ & 50 & $>50$ & $<50$ & 50 & 50 & $>50$ \\
\hline Dimetridazole & 50 & $>50$ & 50 & $>50$ & 50 & $>50$ & 50 & $>50$ & 50 & $>50$ & 20 & 30 & 50 & $>50$ \\
\hline Formalin & 50 & $>50$ & 50 & $>50$ & NT & NT & $<50$ & 50 & 50 & $>50$ & NT & NT & $<50$ & 50 \\
\hline Furacin & 18 & 25 & 15 & 20 & 50 & $>50$ & 18 & 30 & 20 & 20 & 35 & 50 & 12 & 50 \\
\hline Furazolidone & 4 & 8 & 1 & 3 & NT & NT & 3 & 6 & 3 & 5 & 3 & 8 & 2 & 6 \\
\hline Furazolidone $98 \%$ & NT & NT & $<5$ & 5 & 15 & 50 & 5 & 20 & 5 & 10 & 10 & 20 & 5 & 10 \\
\hline Furazolidone, $20 \%$ & NT & NT & NT & NT & NT & NT & 6 & 10 & 15 & 20 & 12 & 15 & 5 & 8 \\
\hline Methylene blue & 35 & 50 & $<40$ & 40 & 20 & 50 & 35 & 40 & 15 & 40 & $<50$ & 50 & 40 & 50 \\
\hline Nalidixic acid & 50 & $>50$ & 35 & $>100$ & 50 & $>100$ & 70 & $>100$ & 25 & $>100$ & 5 & $>100$ & 75 & $>100$ \\
\hline Neomycin sulf. & $<30$ & 30 & 50 & 70 & 40 & 50 & 40 & 90 & 25 & 50 & 60 & 80 & 75 & $>100$ \\
\hline NF200 & 10 & 50 & 5 & 12 & NT & NT & 12 & 50 & 8 & 10 & 15 & 50 & 5 & 10 \\
\hline Nitrofurantoin & 10 & 15 & 5 & 15 & $>25$ & $>25$ & 8 & 10 & 10 & 12 & 10 & 10 & 10 & 12 \\
\hline Nitrofurazone & 2 & 5 & 2 & 5 & 8 & 10 & 2 & 8 & 2 & 5 & 4 & 5 & 2 & 8 \\
\hline Oxacillin $\mathrm{Na}$ & 75 & $>100$ & 75 & $>100$ & 75 & $>100$ & 75 & $>100$ & 75 & $>100$ & 75 & $>100$ & 75 & $>100$ \\
\hline Oxytetracycline & 50 & $>50$ & 50 & $>50$ & 50 & $>50$ & 50 & $>50$ & 50 & $>50$ & 50 & $>50$ & 50 & $>50$ \\
\hline Prefuran & 4 & 5 & 1 & 2 & NT & NT & 4 & 5 & 25 & $>25$ & 4 & 25 & 2 & 3 \\
\hline Prolong & 50 & $>50$ & 50 & $>50$ & 50 & $>50$ & 50 & $>50$ & 50 & $>50$ & 50 & $>50$ & 50 & $>50$ \\
\hline Na nifurstyrenate & 10 & 15 & 3 & 4 & 4 & 10 & 9 & 10 & 15 & 20 & 8 & 40 & 8 & 10 \\
\hline Tylosin tartrate & $<50$ & 50 & $<50$ & 50 & $<50$ & 50 & $<50$ & 50 & $<50$ & 50 & $<50$ & 50 & $<50$ & 50 \\
\hline Vancomycin $\mathrm{HCl}$ & 75 & $>100$ & 75 & $>100$ & 75 & $>100$ & 75 & $>100$ & 75 & $>100$ & 75 & $>100$ & 75 & $>100$ \\
\hline
\end{tabular}


Table 6. Penaeus monodon. Survival rates ( $S_{;} \%$ ), morphological development (D) and behavior (B) of shrimp larvae exposed to selected antibacterial compounds for $24 \mathrm{~h}$. Symbols and abbreviations are: ' significant $\mathrm{p}<0.05, \cdots$ highly significant $p<0.01$; (NT) not tested; (-) normal development; (+) 1 to 3 out of 10 larvae examined had deformities; $(++) 4$ to 6 out of 10 had deformities; $(+++) 7$ to 10 out of 10 had deformities; (IM) incomplete molting; (A) active; (W) weak; (VW) very weak

\begin{tabular}{|c|c|c|c|c|c|c|c|c|c|c|c|}
\hline \multirow[t]{2}{*}{ Chemical } & \multirow{2}{*}{$\begin{array}{l}\text { Assumed } \\
\text { dose range } \\
\left(\mathrm{mg} \mathrm{l}^{-1}\right)\end{array}$} & \multirow{2}{*}{$\begin{array}{c}\text { Dose } \\
\text { tested } \\
\left(\mathrm{mg} \mathrm{l}^{-1}\right)\end{array}$} & \multicolumn{3}{|c|}{ Nauplius } & \multicolumn{3}{|c|}{ Protozoea } & \multicolumn{3}{|c|}{ Mysis } \\
\hline & & & $S$ & $\mathrm{D}$ & B & $\mathrm{S}$ & $\mathrm{D}$ & $\mathrm{B}$ & $\mathrm{S}$ & $\mathrm{D}$ & B \\
\hline Control & & 0 & 89.8 & - & A & 51.5 & - & A & 71.0 & - & A \\
\hline Chloramphenicol & $4->100$ & $\begin{array}{r}5 \\
10 \\
50 \\
100\end{array}$ & $\begin{array}{r}100.0 \\
100.0 \\
93.0 \\
0 \%\end{array}$ & $\begin{array}{l}+ \\
- \\
++\end{array}$ & $\begin{array}{l}\text { A } \\
A \\
W\end{array}$ & $\begin{array}{l}67.8 \\
53.5 \\
53.5 \\
18.5\end{array}$ & $\begin{array}{l}- \\
+ \\
+ \\
+++\end{array}$ & $\begin{array}{l}\text { A } \\
\text { A } \\
\text { W } \\
\text { W }\end{array}$ & $\begin{array}{l}\text { NT } \\
93.0 \\
\text { NT } \\
43.0\end{array}$ & $\begin{array}{l}- \\
\text { IM }\end{array}$ & A \\
\hline Furacin & $40->100$ & $\begin{array}{r}30 \\
50 \\
100\end{array}$ & $\begin{array}{l}\text { NT } \\
86.8 \\
\text { NT }\end{array}$ & + & A & $\begin{array}{c}N T \\
54.0 \\
7.3 \cdots\end{array}$ & $\begin{array}{l}+ \\
+\end{array}$ & $\begin{array}{l}\text { A } \\
W\end{array}$ & $\begin{array}{l}96.4^{\circ} \\
87.4^{\circ} \\
\text { NT }\end{array}$ & $\begin{array}{l}+, I M \\
++\end{array}$ & $\begin{array}{l}\mathrm{A} \\
\mathrm{A}\end{array}$ \\
\hline Nitrofurantoin & $20->50$ & $\begin{array}{l}20 \\
30\end{array}$ & $\begin{array}{l}48.7^{\circ} \\
18.4^{\circ}\end{array}$ & $\begin{array}{l}- \\
-\end{array}$ & $\begin{array}{l}A \\
W\end{array}$ & $\begin{array}{l}56.6 \\
44.5\end{array}$ & $\begin{array}{l}+ \\
+\end{array}$ & $\begin{array}{l}\mathrm{A} \\
\mathrm{A}\end{array}$ & $\begin{array}{l}19.4^{*} \\
15.5^{\circ}\end{array}$ & $\begin{array}{l}+ \\
+, I M\end{array}$ & $\begin{array}{l}A \\
A\end{array}$ \\
\hline Nitrofurazone & $10-20$ & $\begin{array}{r}4 \\
10 \\
16\end{array}$ & $\begin{array}{r}96.4 \\
69.4 \\
6.4\end{array}$ & $\begin{array}{l}+, I M \\
+ \\
+\end{array}$ & $\begin{array}{l}A \\
W \\
W\end{array}$ & $\begin{array}{l}9.0^{\circ} \\
0^{\circ} \\
0^{\circ}\end{array}$ & + & A & $\begin{array}{l}74.0 \\
16.2^{\cdots} \\
8.2^{\cdots}\end{array}$ & $\begin{array}{l}++ \\
+, \mathrm{IM} \\
+\end{array}$ & $\begin{array}{l}\text { A } \\
\text { A } \\
\text { W }\end{array}$ \\
\hline Oxytetracycline & $100->100$ & $\begin{array}{l}100 \\
200\end{array}$ & $\begin{array}{l}98.0 \\
83.0\end{array}$ & $\begin{array}{l}- \\
-\end{array}$ & $\begin{array}{l}\mathrm{A} \\
\mathrm{A}\end{array}$ & $\begin{array}{r}25.5 \\
\mathrm{NT}\end{array}$ & - & W & $\begin{array}{l}78.0 \\
\text { NT }\end{array}$ & - & A \\
\hline Prefuran & $2->25$ & $\begin{array}{r}2 \\
4 \\
10\end{array}$ & $\begin{array}{l}68.5 \\
\text { NT } \\
48.0^{\circ}\end{array}$ & $\begin{array}{l}- \\
++\end{array}$ & $\begin{array}{l}A \\
W\end{array}$ & $\begin{array}{l}69.3 \\
50.5 \\
15.6^{\circ}\end{array}$ & $\begin{array}{l}+ \\
++ \\
+\end{array}$ & $\begin{array}{l}A \\
W \\
W\end{array}$ & $\begin{array}{l}94.0^{\circ} \\
92.0 \\
73.2\end{array}$ & $\begin{array}{l}+ \\
+, \text { IM } \\
\text { IM }\end{array}$ & $\begin{array}{l}\text { A } \\
\text { A } \\
\text { VW }\end{array}$ \\
\hline $\begin{array}{l}\text { Sodium } \\
\text { nifurstyrenate }\end{array}$ & $6-80$ & $\begin{array}{l}10 \\
20 \\
40 \\
50\end{array}$ & $\begin{array}{l}84.0 \\
85.4 \\
62.0 \\
67.3\end{array}$ & $\begin{array}{l}- \\
++ \\
+, \mathrm{IM} \\
+, \mathrm{IM}\end{array}$ & $\begin{array}{l}A \\
A \\
W \\
W\end{array}$ & $\begin{array}{c}56.7 \\
55.3 \\
\text { NT } \\
6.0^{\circ}\end{array}$ & $\begin{array}{l}+ \\
+ \\
+\end{array}$ & $\begin{array}{l}A \\
A \\
W\end{array}$ & $\begin{array}{l}86.6 \\
88.7 \\
89.7 \\
\text { NT }\end{array}$ & $\begin{array}{l}+, I M \\
+ \\
+\end{array}$ & $\begin{array}{l}\text { A } \\
\text { A } \\
\text { A }\end{array}$ \\
\hline
\end{tabular}

bacterial activity in seawater at lower concentrations (15 $\mu \mathrm{g} \mathrm{l}^{-1}$ ) compared to oxytetracycline (inactive at $150 \mu \mathrm{g} \mathrm{l}^{-1}$ ) and chloramphenicol (inactive at $15 \mu \mathrm{g} \mathrm{l}^{-1}$ ) (Ford et al. 1986).

Contrary to popular belief, subinhibitory concentrations do not generate a significant increase in bacterial resistance to the respective drugs (Lorian \& Ernst 1987). Rather, it is the prolonged, repeated or widespread use of an antibiotic that leads to the development of resistance in bacterial populations (Watanabe et al. 1971, Aoki 1974 in Nusbaum \& Shotts 1981, Aoki et al. 1981, 1987). Moreover, rotating the use of several antibiotics may be ineffective because transfer of multiple resistance patterns may occur (Aoki \& Egusa 1971 in Nusbaum \& Shotts 1981, Aoki et al. 1987).

Luminous vibrios enter the larvae primarily through the mouth. They may form plaques in the oral cavity, and also abound in the gut (Lavilla-Pitogo et al. 1990). Thus, any chemical intended for therapy must enter the larva and reach therapeutic tissue levels. One such drug is Furanace (like Prefuran, it has nifurpirinol as its active ingredient) which can cross the gill epithelium of fishes and reach therapeutic tissue levels (Amend \& Ross 1970), presumably due to its small molecular size and ionic charge in water solution (Colorni \& Paperna 1983). However, shrimp larvae could hardly tolerate exposure to the lowest bactericidal dose of Prefuran ( $4 \mathrm{mg} \mathrm{l}^{-1}$ ) based on their weak swimming behavior and morphological deformities.

It appears that a promising drug would be $10 \%$ sodium nifurstyrenate which did not cause morphological deformities in nauplii exposed to a bactericidal dose of $10 \mathrm{mg} \mathrm{l}^{-1}$ for $24 \mathrm{~h}$. However, it caused deformities in older stages of larvae. Vibrio anguillarum and other pathogenic Vibrio species isolated from cultured fishes in Japan have also been found to be sensitive to sodium nifurstyrenate (Ogawa et al. 1982). All Vibrio strains isolated from rotifers (Brachionus plicatilis) were likewise found sensitive to the drug, with MICs at 0.1 to $1.6 \mu \mathrm{g} \mathrm{ml}^{-1}$ (Tanasomwang \& Muroga 1989) which are much lower than values obtained in this study.

Because luminous vibrios effect serious mortalities in shrimp larval rearing systems (Lavilla-Pitogo et al. 1990), it is imperative that measures for their control be developed. Chemical treatment of luminous vibriosis among shrimp larvae is quite limited because of the ineffectiveness of existing and readily available drugs, possible development of resistance in bacteria, human 
health hazard, high toxicity and prohibitive cost of the drugs. The limitations of chemical treatment of luminous bacterial infections among larvae restrict the use of this method of control. It appears that the best method would still be prevention, with particular concentration on rigorous water management and sanitation to prevent the entry of luminous vibrios through the culture water. Further research should also address disinfection of spawners or elimination of the luminous vibrios from spawners which serve as carriers of the pathogens.

Acknowledgements. Several chemicals were donated by Mr J. Alegre of Argent Chemical Laboratories Philippines, Inc., Dr $J$. Hertrampf of BASF Aktiengesellschaft, Dr L. Salvador, and Mr L. Mombay in Tigbauan, Iloila. The SEAFDEC Crustacean Hatchery and the Philippine Aquaculture Specialists, Inc. (AQUASPEC) provided the larvae. We thank Dr A. T. Llobrera and R. Billiones for help during the preliminary part of this study.

\section{LITERATURE CITED}

Amend, D. F, Ross, A. J. (1970). Experimental control of columnaris disease with a new nitrofuran drug, P-7138. Prog. Fish Cult. 32: 19

Aoki, T., Kitao, T., Kawano, K. (1981). Changes in drug resistance of Vibrio anguillarum in cultured ayu, Plecoglossus altivelis Temminck and Schlegel, in Japan. J. Fish Dis. 4: 223-230

Aoki, T., Sakaguchi, T., Kitao, T (1987). Multiple drug-resistant plasmids from Edwardsiella tarda in eel culture ponds. Nippon Suisan Gakk. 53: 1821-1825

Aquacop (1977). Observations on diseases of crustacean cultures in Polynesia. Proc. Wld Maricult. Soc. 8: 685-703

Bell, T. A., Lightner, D. V. (1987). An outline of penaeid shrimp culture methods including infectious disease problems and priority drug treatments. Vet. Human Toxicol. 29 (Suppl. 1): $37-43$

Colorni, A., Paperna, I. (1983). Evaluation of nitrofurazone baths in the treatment of bacterial infection of Sparus aurata and Oreochromis mossambicus. Aquaculture 35 $181-186$

Farkas, J., Olah, J., Szecsi, E. (1982). Antibiotic sensitivity of bacteria isolated from water and fish. Aquacultura Hungarica (Szarvas) 3: 85-92

Responsible Subject Editor: Dr J. E. Stewart, Dartmouth, N.S., Canada
Finegold, S. M., Martin, W. J. (eds.) (1982). Bailey and Scott's Diagnostic Microbiology. The C. V Mosby Company, Philadelphia and Toronto

Ford, L. A., Alexander, S. K., Cooper, K. M., Hanlon, R. T (1986). Bacterial populations of normal and ulcerated mantle tissue of the squid, Lolliguncula brevis. J. Invertebr Path. 48: 13-26

Jones, D. A., Kurmaly, K., Arshard, A. (1987). Penaeid shrimp hatchery trials using microencapsulated diets. Aquaculture 64: 133-146

Lavilla-Pitogo, C. R., Baticados, M. C. L., Cruz-Lacierda, F. R., de la Peña, L. D. (1990). Occurrence of luminous bacterial disease of Penaeus monodon larvae in the Philippines. Aquaculture (in press)

Licop, M. S. R. (1988). Sodium-EDTA effects on survival and metamorphosis of Penaeus monodon larvae. Aquaculture $74: 239-247$

Lorian, V., Ernst, J. (1987). Effects of antibiotics on bacterial structure and their pathogenicity. Path. Biol. 35: 1370-1376

Nusbaum, K. E., Shotts, E. B. (1981). Action of selected antibiotics on four common bacteria associated with diseases of fish. J. Fish Dis. 4: 397-404

Ogawa, S., Hatai, K., Yasumoto, S., Hirakawa, E., Yasunaga, N. (1982). Drug sensitivity of various drug-pathogenic bacteria isolated from cultured fish in Nagasaki Prefecture recently. Bull. Nagasaki pref. Inst. Fish. 8: 91-100

Pitogo, C. R. (1988). Isolation and identification of luminous bacteria causing mortalities in Penaeus monodon hatcheries in Panay. SEAFDEC Asian Aquaculture 10: 9

Poupard, C. J. (1978). Therapy of fish diseases. In: Roberts, R. J. (ed.) Fish pathology. Bailliere Tindall, London, p. $268-275$

SIGMA (1989). Biochemical and Organic Coumpounds for Research and Diagnostic Clinical Reagents (Catalogue). SIGMA Chemical Company, St. Louis, Missouri

Sunaryanto, A., Mariam, A. (1986). Occurrence of a pathogenic bacteria causing luminescence in penaeid larvae in Indonesian hatcheries. Bull. Brackishwater Aqua. devl Center 8: 64-70

Tanasomwang, V., Muroga, K. (1989). Effects of sodium nifurstyrenate and tetracycline on the bacterial flora of rotifers (Brachionus plicatilis). Fish Pathol. 24: 29-35

Watanabe, T., Aoki, T., Ogata, Y., Egusa, S. (1971). R factors related to fish culturing. Ann. N.Y. Acad. Sci 182: $383-410$

Williams, R. R., Lightner, D. V. (1988). Regulatory status of therapeutants for penaeid shrimp culture in the United States. J. Wld Aquacult. Soc. 19: 188-196

Manuscript first received: February 19, 1990

Revised version accepted: June 18, 1990 